\title{
Feature Preserving Mesh Simplification Using Feature Sensitive Metric
}

\author{
Jin $\mathrm{Wei}^{1}$ (魏 瑨), Student Member, CCF, ACM, and Yu $\mathrm{Lou}^{2}$ (楼 宇), Student Member, ACM \\ ${ }^{1}$ Tsinghua National Laboratory for Information Science and Technology \\ Department of Computer Science and Technology, Tsinghua University, Beijing 100084, China \\ ${ }^{2}$ Department of Computer Science, Stanford University, Stanford, 94305, U.S.A.
}

E-mail: wei-j07@mails.tsinghua.edu.cn; yulou@stanford.edu

Received April 20, 2009; revised March 18, 2010.

\begin{abstract}
We present a new method for feature preserving mesh simplification based on feature sensitive (FS) metric. Previous quadric error based approach is extended to a high-dimensional FS space so as to measure the geometric distance together with normal deviation. As the normal direction of a surface point is uniquely determined by the position in Euclidian space, we employ a two-step linear optimization scheme to efficiently derive the constrained optimal target point. We demonstrate that our algorithm can preserve features more precisely under the global geometric properties, and can naturally retain more triangular patches on the feature regions without special feature detection procedure during the simplification process. Taking the advantage of the blow-up phenomenon in FS space, we design an error weight that can produce more suitable results. We also show that Hausdorff distance is markedly reduced during FS simplification.
\end{abstract}

Keywords mesh simplification, feature preserving, feature sensitive (FS) metric

\section{Introduction}

Simplification of meshes with arbitrary topology plays an important role in digital geometry processing. It is a basis for multiresolution representation, data reduction, real-time rendering or even as a pre-processing stage for segmentation and data fitting. 3D acquisition techniques such as laser scanning produce more and more complex polygonal models, but over millions of triangles are not always needed in most graphics systems. Many applications require a trade-off between efficiency and realism to achieve interactivity. The issues become to reduce complexity of triangular mesh on the premise of less deviation to the original model.

The early methods including clustering and decimation may preserve neither topology nor visual quality ${ }^{[1]}$. Garland and Heckbert ${ }^{[2]}$ provided a Quadric Error Metric (QEM) based edge-contraction algorithm that generally produces better result. It has been widely used due to its efficiency and accuracy, and there exist quite a lot of improved implementations in different situations.

The key of QEM algorithm is to find an error metric as the cost function at each vertex, and the vertex pair which has the minimum cost is contracted at each iteration step. However, because this error measurement is solely based on the Euclidian distance between geometric positions, simplification may preserve geometric features only to a certain extent, i.e., it just produces slightly more triangles in feature regions than planar ones. Consider a parameterization to a feature sensitive space, simplified mesh will get sparse at features but dense at smooth region. An intuitive expectation is to provide a scheme that automatically retains more patches for features. Furthermore, we expect simplified control points near the original feature line. Based on similar ideas, much work has been done to improve the retaining rate of the geometric details by introducing new error arguments.

To obtain new error metric related to features, many researches employ a recognition procedure, or simply re-computing and adding several different types of error matrices to form a single cost function at every iteration step ${ }^{[3-5]}$. It may cause more complexity during the simplification process.

Generally, human visual system is more sensitive to the curved parts, including the boundary, crease, corner etc. Therefore, it is natural to use the changing degree of surface unit normal such as curvature, to quantize the local feature sensitivity. Notice that position and normal information are individual to a point in three-dimensional Euclidian space. Based on the idea

Regular Paper

This work was supported by the National Basic Research 973 Program of China (Grant No. 2006CB303106), the National Natural Science Foundation of China (Grant Nos. 60673004, 90718035) and the National High Technology Research and Development 863 Program of China (Grant No. 2007AA01Z336).

C) 2010 Springer Science + Business Media, LLC \& Science Press, China 
of image manifold ${ }^{[6]}$, Lai et al. ${ }^{[7]}$ map each point in $\mathbb{R}^{3}$ into a six-dimensional image with a non-negative $w$ indicating feature sensitivity, so that a point in FS space will be in the form of $(\boldsymbol{p}, w \boldsymbol{n})$. [8] also proves that the area size in FS space is directly related to the local curvature properties. In addition, mapping to FS space could produce a blow-up phenomenon at sharp features such as creases and corners according to the user requirements. Detected feature lines will be expanded to the surface area in $\mathbb{R}^{6}$ to adjust the mesh density in FS space. Thus, using FS metric, it can naturally increase the quality in feature regions, without the necessity of explicitly detecting sharp features (Fig.1). The usefulness of FS metric has been demonstrated in the applications of remeshing ${ }^{[7]}$, mesh segmentation ${ }^{[9]}$, surface fitting $^{[8]}$, and feature extraction and classification ${ }^{[7]}$.

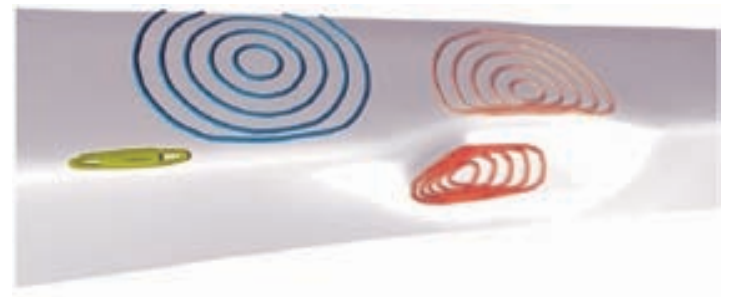

Fig.1. Distance isolines in feature sensitive space (this figure is from [7]).

In this paper, we present a novel mesh simplification based on a feature sensitive metric, for efficiently simplifying polygonal meshes with a new quadric error function defined in feature sensitive space. This method extends classical QEM to a constraint highdimensional space with specific rule for singular situations. Experimental results demonstrated that our approach can naturally allocates more patches to the

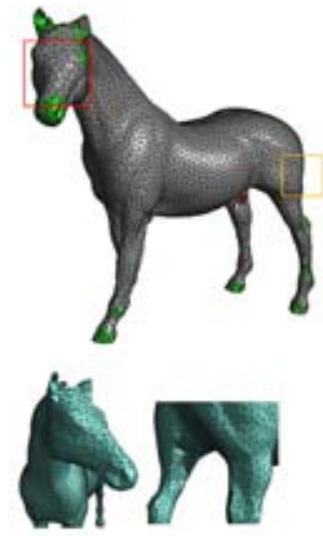

(a)

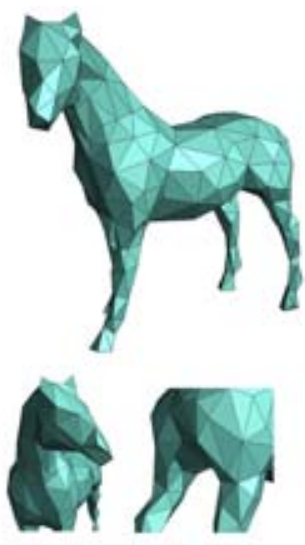

(b)

feature regions, without complicated procedure for feature detection. We also proposed a weight scheme to resemble the blow-up phenomenon that is described in [8]. With the proper preferences, this algorithm will strongly improve the visualization quality with lower Hausdorff distances.

In Section 2 we review the previous work about mesh simplification, and describe their differentia to our algorithm. Then the new scheme for our FS mesh is discussed in Section 3. Section 4 presents the optimization method to preserve singular situations at boundaries and blow-up. Experimental results are shown in Section 5 and the conclusions and remarks on future work are given in Section 6 .

\section{Related Work}

To preserve features during the simplification, we have chosen to design a pair-contraction based method. The main issue then becomes to define cost priority and selection of optimizing target position that produce minimum error.

\section{- $Q E M$}

Garland and Heckbert ${ }^{[2]}$ defined the error metric as the sum of squared distance from a given point to its corresponding plane. This can be easily converted into a quadrics matrix form. Therefore the error function was constructed as: $\eta(\boldsymbol{v})=\boldsymbol{v}^{\mathrm{T}} Q \boldsymbol{v}$. Positions that have the smallest $\eta(\boldsymbol{v})$ then become contraction target point for a pair. In addition, $\eta(\boldsymbol{v})$ should be sorted ascending into a heap so as to determine the contraction order.

\section{- Preserving Features}

QEM method can preserve position information well with efficiency than the previous simplification method. However, the error metric of QEM is based only on positions but not for the curvature or the variation of

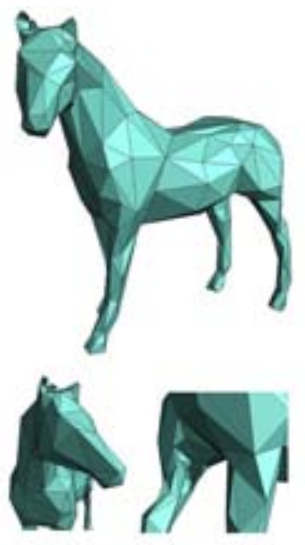

(c)

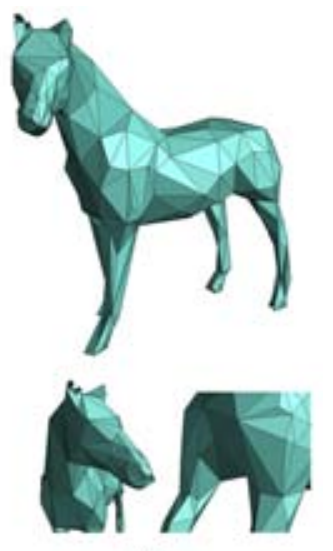

(d)

Fig.2. Horse model (10024 tri. to 596 tri.) with green (or red) colored feature region. (a) Input mesh. (b) QSlim result. (c) (d) Our FS method without and with blow-up. The corresponding zoom in viewer are under them, $\omega=0.03$. Obviously (c) produces more reasonable result than (b), and (d) preserves more detail for the head rather than retain the triangles on the leg and joints. 
colors and texture. Having taken note of this, a lot of work has been proposed over how to enrich the definition of the error metric with the other information. Garland and Heckbert ${ }^{[2]}$ introduce surface attribute and convert the mesh into a high-dimensional space that is constructed by the positions and colors, then they measure the distance from an $n$-dimensional point to corresponding $n$-D hyperplane. Hoppe ${ }^{[3]}$ extended this method to a higher-dimensional space that may combine information including normal, color and texture coordinates. He projected each point into attribute space for measurement. This is equivalent to construct a combined error metric. In addition, Cohen et al. ${ }^{[10]}$ designed a texture coordinate related simplification approach that reduces the distortion for texture mapped models. Since then, the research about feature preserve contraction has become to propose a new combination of the error matrices, such as [4-5]. Moreover some direct methods such as [11] are proposed to preserve feature as the user specifies by UI input. Garland ${ }^{[12]}$ summarized the method based on high-dimensional space and extends to arbitrary manifold. In addition, attribute analysis is also introduced in order to preserve pre-detected features such as ${ }^{[4,13-14]}$. Based on the exactly extracted feature lines, these methods extended QEM into a weighted quadric metric, vertices close to feature line get a large weight so that they are hard to eliminate. Moreover, rather than finding a local minimization in $[2-3,12],[15]$ proposed a variational approach to computing the simplified mesh under global optimization method, in which the metric in [2] was extended to processing mesh normals. This method is good to capture anisotropy and also to reduce the geometric errors.

\section{Simplification in FS Space}

In this section, we will discuss the details of our proposed algorithm. The overall pipeline of the algorithm is first presented. Quadric error matrix extended in FS space is then discussed in detail. We finally give an algorithm to compute the optimized position. Since position and normal at each vertex is not freely separated, we introduce an optimization based algorithm to find the optimal vertex position in replace of an contracted edge.

\subsection{Algorithm Overview}

QEM based method is generally a "SelectionContraction" iteration process. In three-dimensional Euclidian space, quadric metric that indicate the sum of squared distance from a space point to its correlative adjacent plane can be converted into a $4 \times 4$ symmetric matrix under homogeneous coordinates. It is in fact the discrete form of TDM (Tangent Distance Minimization) from the space point to the vertex of the current valid pair.

In FS space, we describe a six-dimensional vector as $\boldsymbol{v}_{i}=\left(\boldsymbol{p}_{i}, w \boldsymbol{n}_{i}\right)$, where $\boldsymbol{p}_{i}$ and $\boldsymbol{n}_{i}$ indicate its position and normal in $\mathbb{R}^{3}$ respectively, while $w$ is the feature sensitivity weight as defined in the previous section. Its error matrix $\boldsymbol{Q}_{i}$ is then extended to a $6 \times 6$ symmetric matrix. Following the principle proposed in [2], the matrix form of discrete squared tangent-point distance in FS space

$$
\varepsilon_{i}(\boldsymbol{v})=\left(\boldsymbol{v}-\boldsymbol{v}_{i}\right)^{\mathrm{T}} \boldsymbol{Q}_{i}\left(\boldsymbol{v}-\boldsymbol{v}_{i}\right)
$$

is defined to be the contraction error for each vertex $\boldsymbol{v}_{i}$, and the cost of the $k$-th contraction $\left(\boldsymbol{v}_{i}, \boldsymbol{v}_{j}\right) \rightarrow \overline{\boldsymbol{v}}$ is similarly chosen to be $\xi_{k}(\overline{\boldsymbol{v}})=\varepsilon_{i}(\overline{\boldsymbol{v}})+\varepsilon_{j}(\overline{\boldsymbol{v}})$.

In order to preserve feature more precisely, the best choice to derive contraction target point is to find an optimal vertex $\overline{\boldsymbol{v}}$ that minimizes the error function $\xi_{k}(\overline{\boldsymbol{v}})$. Notice that $\xi_{k}(\overline{\boldsymbol{v}})$ is now an energy function defined in FS space, the main difference to $\mathbb{R}^{3}$ is that optimization in FS space is in fact a constrained optimization problem. In Subsection 3.2, we introduce an efficient constraint iteration scheme to fast obtain the optimized target point under FS error metric.

Now we could give a summary of our FS simplification algorithm as follows. Selection of valid pairs have been done during initialization and the selection rules are similar as described in [2]. As we map unit normal vector for each vertex with a non-negative $w$, in order to produce uniform effects for this quantity on different models, we simply fitted all models into a unit cube before FS mapping, and rescale back after the simplification. In this paper, we set $w=0.03$ expect for specific statement.

Input: polygonal mesh in FS space.

Output: simplified mesh with more triangles remaining in the feature regions.

Algorithm:

1) For each vertex, compute the $6 \times 6$ FS error matrix $Q_{i}$.

2) For each valid pair, compute the target point $\left(\boldsymbol{v}_{i}, \boldsymbol{v}_{j}\right) \rightarrow \overline{\boldsymbol{v}}$ that minimizes the constrained cost function $\xi_{k}(\overline{\boldsymbol{v}})=\varepsilon_{i}(\overline{\boldsymbol{v}})+\varepsilon_{j}(\overline{\boldsymbol{v}})$; push every valid pairs into a minimum-heap according to the quantity of $\xi_{k}(\overline{\boldsymbol{v}})$.

3) Contract a vertex pair which is the top element in the heap, update the error for each new adjacent vertices as 2 , iterate until the target amount of triangles is achieved.

4) Post cleaning (Optional).

Note that the input mesh is a polygonal mesh mapped to FS space. It may have blow-up phenomenon 
about feature line ${ }^{[8]}$. This may cause some singular problem during the process because they have identical position vector in $\mathbb{R}^{3}$. The treatments for blow-up region are discussed in Section 4.

\subsection{Derive Quadric Matrix in FS Space}

Definition of the cost function is a pacing factor for visual effect. It is noticed that a mesh in FS space is actually a 2-manifold embed in $\mathbb{R}^{6}$. FS space is therefore a non-free form space that is constrained by the geometric properties and topology in three-dimensional Euclidian space. Because the input model is existing in $\mathbb{R}^{3}$ in essence, operations in FS space must satisfy the space restriction. Error measurements is then defined from $\mathbb{R}^{6}$ to original $\mathbb{R}^{3}$. Previous works have chosen to use the discrete approximation of the tangent plane at a mesh vertex. Here we map the planes of the triangles that meet at the original vertex in $\mathbb{R}^{3}$ onto a 2 -d hyperplane embed in $\mathbb{R}^{6}$ to approximate the corresponding tangent space. The contraction cost is then defined by the squared distance between the space point and that 2-d hyperplane.

As we have three given points lying on the triangle that are mapped from $\mathbb{R}^{3}$, one can always get two edge vectors $\boldsymbol{e}_{1}$ and $\boldsymbol{e}_{2}$ in $\mathbb{R}^{6}$. Then, for a six-dimensional element $\boldsymbol{v}$, evaluating the distance to the 2-hyperplane which determined by this triangle is essentially equivalent to finding the nearest point $\boldsymbol{v}_{\text {min }}$ in the linear subspace $\boldsymbol{W}$ that is spanned by $\boldsymbol{e}_{1}$ and $\boldsymbol{e}_{2}$ :

$$
d_{i}^{2}=\min \left\|\boldsymbol{v}-\boldsymbol{v}_{t}\right\|^{2}, \quad \boldsymbol{v}_{t} \in \boldsymbol{W}
$$

where $\boldsymbol{v}_{t}$ is an arbitrary vertex in $\boldsymbol{W}$. To this end, we aimed to finding a vector $\boldsymbol{v}_{\perp}=\boldsymbol{v}-\boldsymbol{v}_{\min }-\boldsymbol{v}_{i}$ which is orthogonal to the linear subspace $\boldsymbol{W}$ with its origin at $\boldsymbol{v}_{i}$. Let us define a $2 \times 6$ matrix $\boldsymbol{A}=\left[\begin{array}{ll}\boldsymbol{e}_{1} & \boldsymbol{e}_{2}\end{array}\right]^{\mathrm{T}}$ and local coordinates $\boldsymbol{x}_{\text {min }}$ on the basis of the subspace, i.e., $\boldsymbol{v}_{\min }=\boldsymbol{A}^{\mathrm{T}} \boldsymbol{x}_{\min }+\boldsymbol{v}_{i}$. We could now set an equation as:

$$
\boldsymbol{A}\left(\boldsymbol{v}-\boldsymbol{A}^{\mathrm{T}} \boldsymbol{x}_{\min }-\boldsymbol{v}_{i}\right)=0
$$

Solving (3) w.r.t. $\boldsymbol{x}_{\min }$, we derived that

$$
\boldsymbol{v}_{\min }=\boldsymbol{A}^{\mathrm{T}}\left(\boldsymbol{A} \boldsymbol{A}^{\mathrm{T}}\right)^{-1} \boldsymbol{A}\left(\boldsymbol{v}-\boldsymbol{v}_{i}\right) .
$$

So that

$$
\begin{aligned}
\boldsymbol{v}_{\perp} & =\boldsymbol{v}-\boldsymbol{v}_{\min }-\boldsymbol{v}_{i} \\
& =\left(\boldsymbol{I}-\boldsymbol{A}^{\mathrm{T}}\left(\boldsymbol{A} \boldsymbol{A}^{\mathrm{T}}\right)^{-1} \boldsymbol{A}\right)\left(\boldsymbol{v}-\boldsymbol{v}_{i}\right) \\
& =\boldsymbol{K}\left(\boldsymbol{v}-\boldsymbol{v}_{i}\right) .
\end{aligned}
$$

Obviously, $\boldsymbol{K}$ is a $6 \times 6$ symmetric matrix. Moreover, we also noticed that $\boldsymbol{K}$ is implicitly comprised of the Moore-Penrose inversion of $\boldsymbol{A}$. Therefore, $\boldsymbol{K}$ can be rewritten as $\boldsymbol{K}=\boldsymbol{I}-\boldsymbol{A}^{+} \boldsymbol{A}$, such a matrix is idempotent:

$$
\boldsymbol{K}^{2}=\boldsymbol{K}^{\mathrm{T}} \boldsymbol{K}=\boldsymbol{K}
$$

The squared distance from an arbitrary vertex $\boldsymbol{v}$ in FS space to the subspace $\boldsymbol{W}$ is now established as:

$$
\begin{aligned}
d_{i}^{2} & =\boldsymbol{v}_{\perp}^{2} \\
& =\left(\boldsymbol{v}-\boldsymbol{v}_{i}\right)^{\mathrm{T}} \boldsymbol{K}^{\mathrm{T}} \boldsymbol{K}\left(\boldsymbol{v}-\boldsymbol{v}_{i}\right) \\
& =\left(\boldsymbol{v}-\boldsymbol{v}_{i}\right)^{\mathrm{T}} \boldsymbol{K}\left(\boldsymbol{v}-\boldsymbol{v}_{i}\right) .
\end{aligned}
$$

Thus the error metric for $\boldsymbol{v}_{i}$ can be easily derived in an additional form:

$$
\begin{aligned}
\varepsilon_{i}(\boldsymbol{v}) & =\sum d_{i}^{2} \\
& =\sum\left(\boldsymbol{v}-\boldsymbol{v}_{i}\right)^{\mathrm{T}} \boldsymbol{K}_{i}\left(\boldsymbol{v}-\boldsymbol{v}_{i}\right) \\
& =\left(\boldsymbol{v}-\boldsymbol{v}_{i}\right)^{\mathrm{T}} \sum \boldsymbol{K}_{i}\left(\boldsymbol{v}-\boldsymbol{v}_{i}\right) \\
& =\left(\boldsymbol{v}-\boldsymbol{v}_{i}\right)^{\mathrm{T}} \boldsymbol{Q}_{i}\left(\boldsymbol{v}-\boldsymbol{v}_{i}\right)
\end{aligned}
$$

where $\boldsymbol{Q}_{i}$ is set to be the error matrix of $\boldsymbol{v}_{i}$. Similar to the previous works, we suggest using homogenous form to represent $\varepsilon_{i}$, thus the updated error of new contract point can simply become an additional form.

\subsection{Contraction Point Optimization}

Edge contraction can be treated as an iteration process. Although we can simply choose the target position as $\boldsymbol{v}_{i}, \boldsymbol{v}_{j}$ or their midpoint, to preserve geometric details as much as possible, the best choice is to estimate an optimized location that minimizes the six-dimensional quadric error with the geometric constraint in $\mathbb{R}^{3}$.

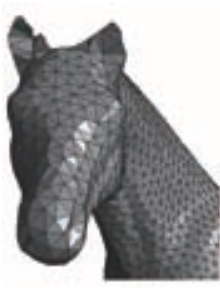

(a)

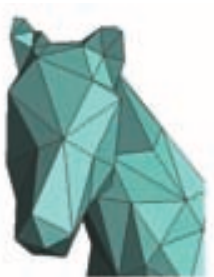

(b)

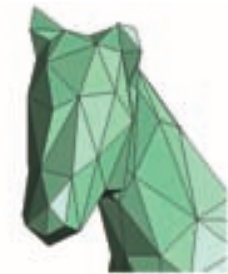

(c)
Fig.3. Comparison with non-constraint optimization in FS space. (a) Original mesh. (b) Our method. (c) Modified QEM $\operatorname{method}^{[12]}$

Note that in FS space, vertex is combined by its position and weighted normal in $\mathbb{R}^{3}$. Once a position in $\mathbb{R}^{3}$ has been set, the attached normal vector will be uniquely determined by its local geometry. Due to this restriction, solving the minimization problem directly in FS space without constraint may lead to unexpectable result (see Fig.3). Therefore we propose an iteration scheme with linear search and an initial guess $\boldsymbol{v}_{i}=\left(\boldsymbol{p}_{i}, \omega \boldsymbol{n}_{i}\right)$ to derive a constrained optimization target point in FS space. The flowchart of this optimization procedure is shown in Fig.4. 


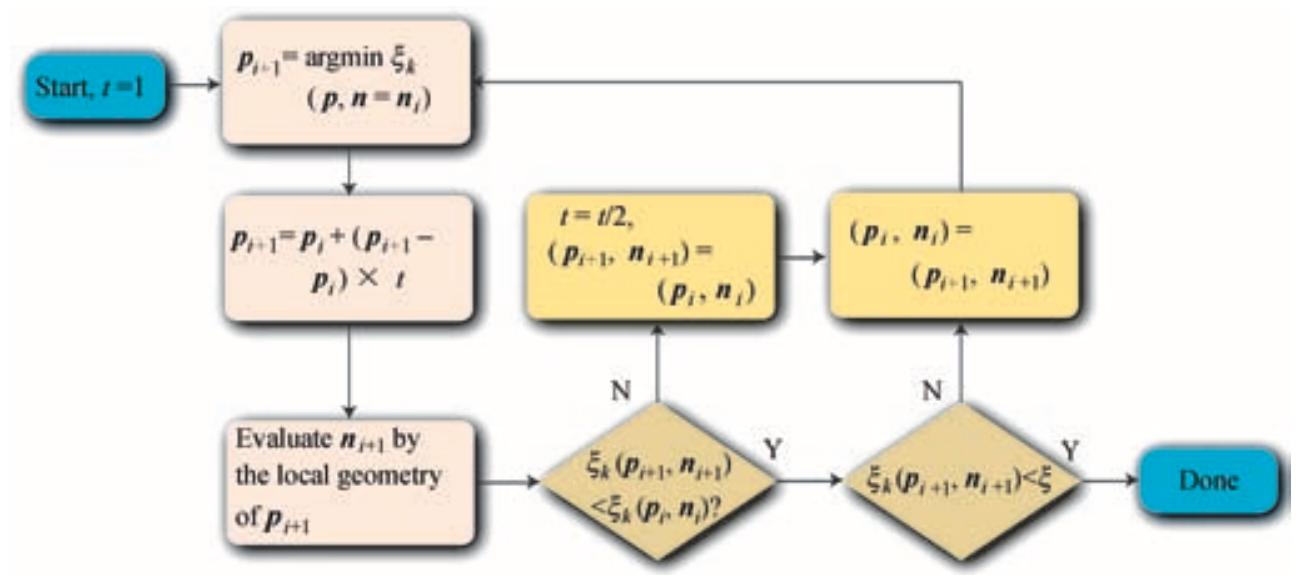

Fig.4. Flowchart of the constrained optimization procedure for deriving target contraction point.

In case we obtain an optimized position $\boldsymbol{p}_{i}^{\prime}$ with a fixed normal $\boldsymbol{n}_{i}^{\prime}$, the corresponding normal $\boldsymbol{n}_{i}^{\prime}$ for this local optimization position could be derived by its local geometry.

Notice that as the step factor $t$ can strictly reduce the quadric error during each iteration step, the optimization is guaranteed to be a convergent procedure. To assign an initial value, we get optimized $\boldsymbol{p}_{i+1}$ under fixed $\boldsymbol{n}_{\text {mid }}$ that derived from the average of two original normals. The corresponding normal vector $\boldsymbol{n}_{i+1}$ is then evaluated by the revised local geometry. According to Subsection 3.1, cost function is defined to be a quadratic form, so that searching between initial point and optimized point is a linear problem. In our experiments, we have found out that most iteration will be done within $2 \sim 4$ times. Fig. 5 shows the results using linear search iteration in different times, searching within 4 times provides acceptable effects.

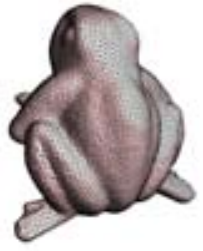

(a)

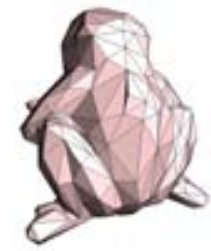

(b)

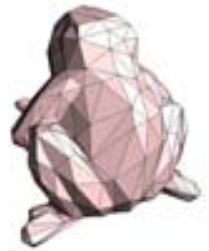

(c)
Fig.5. Target point optimization with 1-time (see (b)) and 4time (see (c)) iterations for each new vertex. (c) produces more suitable result.

\section{Additional Details to Geometric Optimization}

In this section, we give further details to some steps of the pipeline, including dealing with blow-up regions, and the post cleaning operation.

\subsection{Error Weights for Blow-Up Regions}

It is claimed that the input of our algorithm is a polygonal mesh in FS space. In the theory of featuresensitive geometry processing, one can generate blowup regions for sharp feature when the inner product of two face normals is greater than a user defined threshold, so as to possess further area in parameter domain ${ }^{[8]}$. Such blow-up points which have identical positions but dissimilar normal directions in $\mathbb{R}^{6}$ are essentially the same in the three-dimensional Euclidian space. Simplification without special rules may result in unexpectable mistake about feature region. As described in [8], blow-up phenomenon expand the area of features in parameter domain, so that the result of parametrization is well improved. However, as the more we assign feature sensitivity, the more points would be generated in FS space. In Fig.6, blow-up vertices explicitly decompose the variation of the normal direction

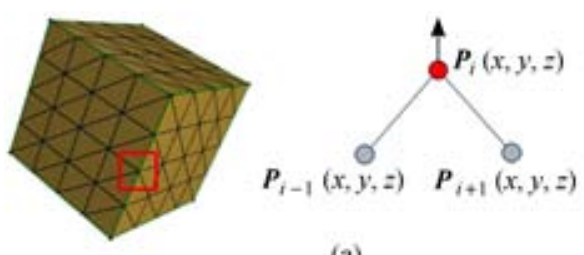

(a)
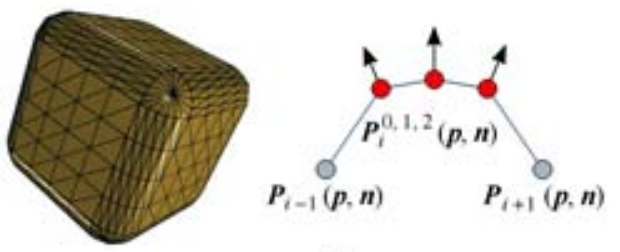

(b)

Fig.6. The blow-up phenomenon at sharp edges and corners. (a) Original mesh in $\mathbb{R}^{3}$. (b) Projection of the corresponding mesh in $\mathbb{R}^{6}$. Normal vectors are set to be the face normals on the two sides of the edge and the interpolation between them. 
on creases and corners. It means that vertices in feature regions in FS space have tiny difference only at the coefficients related to normal direction. When a vertex on sharp feature is decomposed into several new points in FS space, features will get flatter than their preimage in $\mathbb{R}^{3}$, so that contraction error in blow-up regions will be extremely small. This indicates that sharp features which have been blown will get top priority in the error heap. To avoid this, the number of blow-up vertex have to be constrained. We have found out that most feature vertices can at most be decomposed into two or three $6 \mathrm{D}$ points, and the improvement under this strategy could be ignored. This may lose the advantages of both the error metric in FS space and blow-up phenomenon.

For this reason, a specific rule that simulates the effect of blow-up scheme is designed. The basic idea is to change the contraction cost function into a weighted quadric error rather than veritably expands the features into several blow-up points which are identical in $\mathbb{R}^{3}$. The weighted quadric error is then defined as: $\xi_{k}^{\prime}=\eta_{k} \xi_{k}$ where $\eta$ denotes the number of vertices that the current vertex will be expanded to. We firstly launch a shrink-back procedure during initialization to ensure there is no degenerated edge in $\mathbb{R}^{3}$. The blow-up weight is then directly inherited from the blow-up rule in [8] as follows.

1) If a vertex $\boldsymbol{v}$ is neither labeled as edge nor corner vertex, we simply set $\eta_{i}=1$.

2 ) If $\boldsymbol{v}$ lies on the edge which the angle deviation $\theta$ of normal vector on either side of the edge is larger than a threshold value $\theta_{E}$ (we use $\pi / 4$ for all models in this paper), this vertex is treated as edge vertex. The corresponding weight is assigned as:

$$
\eta_{i}=\left\lceil\frac{\theta}{\theta_{t}}\right\rceil+1
$$

where " $\lceil\cdot\rceil$ " denotes the ceil operation, $\theta_{t}$ is an userspecified step factor means the mesh density in blow-up region.

3) If there are at least three sharp edges meeting at a vertex, this vertex is treated as a corner. Moreover, a cone-like vertex is also labeled as corner if the sum of the angle of one-ring neighboring triangles is less than $2 \pi \cos \theta_{\mathrm{C}}$, where $\theta_{\mathrm{C}}$ is the corner threshold, we use $\pi / 12$ in this paper. Blow-up weight for corners is then defined as:

$$
\eta_{i}=\sum \eta_{k}+1
$$

where $\eta_{k}$ is the weight of the $k$-th neighbor vertex of the corner $\boldsymbol{v}$. For simplicity, we do not allow the existence of the two adjacent corners. Once it occurs, we use a dummy point between them and simply set it as an edge point.
According to the above rules, $\Sigma \eta_{i}$ is obvious with the same amount of vertices at the blow-up region in FS space. So this rule will not lose the significance of blowup phenomenon. To ensure that contraction point is a local minimum under FS error metric, blow-up weights should not be taken into account in the optimization. In other words, $\eta$ only influences the priority of the elements in error heap.

The rule for updating error cost of contraction point $\overline{\boldsymbol{v}}$ is also improved. For a vertex pair with at most one blow-up vertex, new weight is set to be the average of $\eta_{i}, \eta_{j}$, so that new error cost equivalent to the general cases. For a pair containing two vertices that the corresponding weights are all greater than 1 , new weight for target point is updated to $\eta_{i}+\eta_{j}$, so that error cost actually becomes $\left(\eta_{j}+\eta_{j}\right)\left(\xi_{i}+\xi_{j}\right)$, meaning that when an edge containing two feature vertices is contracted, the optimized contraction position is an abstraction of the current feature region, so it should be more difficult to simplify.

Although this procedure extract feature naively, we found this strategy gives further improvement to FS metric based method. Results in Fig.2 demonstrates the effectiveness of this method.

\subsection{Preserving and Post Cleaning}

Open boundaries require additional constraint during the optimization process. For a contraction containing boundary points $\boldsymbol{v}_{\mathrm{b}}$, linear search for optimization point is constrained in the plane that is spanned by two boundary edges intersecting at $\boldsymbol{v}_{\mathrm{b}}$. For simplicity, we search the optimized boundary point along the boundary line which consists of $\boldsymbol{v}_{\text {bleft }}, \boldsymbol{v}_{\mathrm{b}}$ and $\boldsymbol{v}_{\text {bright }}$. We test face-flipping after target point optimization. In case that flipping occurred, we use a penalty multiple to
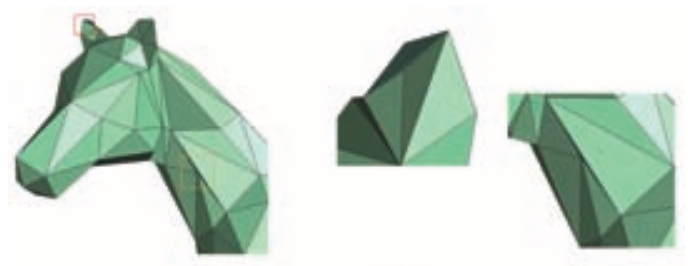

(a)
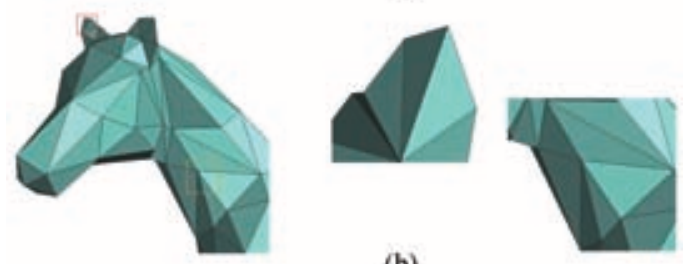

(b)

Fig.7. Edge-flipping to remove near-degenerated triangles. (a) Before edge-flipping. (b) After edge-flipping. The amount of faces is not changed after the flipping process. 
increase their contraction cost. In our experiments, we always use 3 for penalty.

Because this algorithm is based on a feature sensitive metric, sharp features will hold more triangular patches but less triangles to represent planar regions. In many cases, remaining triangles get narrower than another QEM based method. For very few triangles that consist of planar points and near-feature points, it may become narrow or degenerated triangles through the optimization under FS error metric. Consider that narrow triangles may give instabilities to the follow-up processing, we employ an edge-flipping (as Fig.7) treatment to remove degenerated triangles if it satisfies the inequality as:

$$
\left|e d g e_{k}+e d g e_{(k-1)}\right|-\left|e d g e_{(k+1)}\right|<\tau
$$

where $k=0,1,2, \tau$ is a small positive floating number less than 1 (we set as $10^{-4}$ ). Obviously, this treatment does not alter the amount of remaining faces.

\section{$5 \quad$ Results and Discussions}

We have proposed a feature sensitive simplification approach running in FS space. We have implemented this method to investigate with widely used quadric error based simplification framework, QSlim. We compare the results form the visual qualities and statistical analysis of the produced errors (in Fig.8). All test models has distinct feature regions including creases, corners and boundaries as in Figs. 2, 9, 10 and 11, these results demonstrated our method can preserve feature more precisely.

For a simplified mesh, remaining vertices on the planar regions can be treated as redundancy. We have the first impression with Fig.9 that our algorithm retains more patches on the feature region to eliminate the redundant information. To quantize this ability, many criteria can be met, such as [16-17]. In this paper we just use the well-known two-sided Hausdorff distance measurement tool, Metro, as designed in [17] for evaluating mesh quality, to compare the similarity between simplified and original models. In addition, because our algorithm is defined in FS space, we also measure a six-dimensional Hausdorff distance that is defined to be the Euclidian distance from an FS space point to a embedded two-dimensional triangle patch, in order to deal with the effectiveness of error optimization in FS space. To avoid ambiguities we call the Hausdorff distance defined in $\mathbb{R}^{3}$ and $\mathbb{R}^{6}$ as geometric error and FS error (or distance) respectively.

Fig.8 shows some quantified results during experiments with simplification of Lucy model. We observed that FS metric based method produces a significant improvement on geometric error than QSlim. That is, as the traditional quadric errors are not defined in a feature-isotropic space, distortions may be caused when a large data reduction occurred. If we apply the algorithm to simplify massive meshes, tiny errors will accumulate with the increasing of reduction percentage during the simplification process (see Fig.8(a)). Although we calculate optimized solution for contraction at each step, this may still introduce some adverse effects on the visual quality. We know that Hausdorff distance defined in $\mathbb{R}^{3}$ indicates max geometric error between two given models, and large errors are caused when the edge containing feature point is collapsed. Intuitively, human vision system is sensitive to contours and sharp regions. As shown in Fig.8(d), our method preserves the contour of Lucy model and then naturally assigns more triangular patches on the regions with large normal variations. This effect is useful especially when we take a large reduction rate. For this reason, FS method provided preferable results, and, as more feature points are retained, geometric error is greatly reduced compared with QSlim as shown in Fig.8(a).

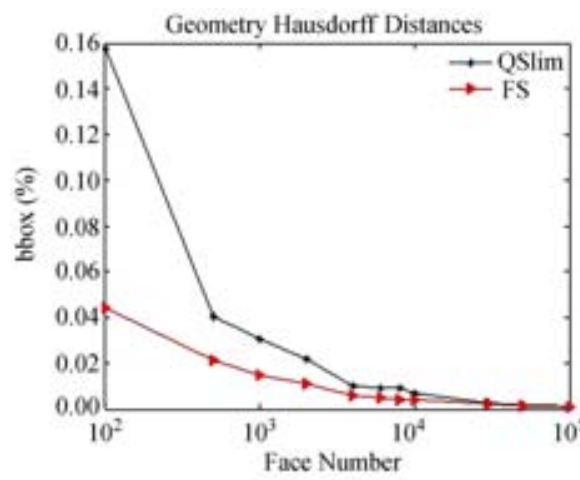

(a)

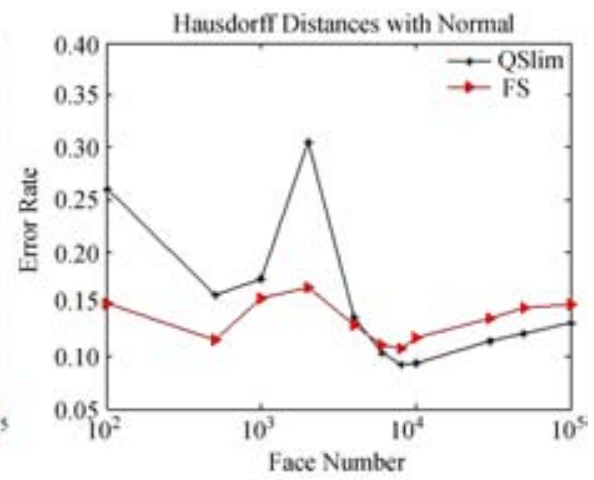

(b)

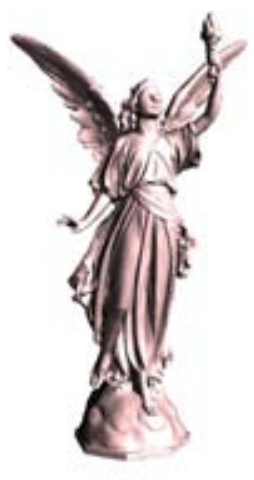

(c)

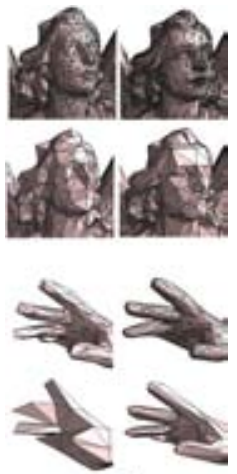

(d)

Fig.8. Quantized produced errors during simplification of Lucy model (c). Errors are classified into two categories as Hausdorff distances in $\mathbb{R}^{3}$ (a) and $\mathbb{R}^{6}$ (b). (d) Zoom in image of details on the simplified models using QSlim (left) and FS (right). Our method gets high visual quality on the details. 
Moreover, we also interested in the Hausdorff distances in FS space. In Fig.8(b), we found that statistical results did not provide remarkable contrast in different stages, but FS method got a steadier error curve and is well constrained under $20 \%$. This is reasonable because the optimization discussed in Section 3 did not make a globally optimized solution, but a constrained local minimum. However, as these constrained-optimized points are derived with an userspecified threshold value uniformly, the overall FS error can be kept within a bound.

In another similar result shown in Fig.9(a), we found that FS method provides a dense approximation for facial features but large triangles on the planar regions like forehead and cheeks. Fig.9(b) also shows that more patches are assigned and retained on the sharp features, but fewer samples are remained on the planar regions under the same reduction rate. This effect is useful for a smooth shading effect on important regions.

In Subsection 4.1, we described the benefits of blowup phenomenon to parametrization. Here we discuss the effects of this strategy in mesh simplification. As we mentioned, blow-up strategy is a naive feature extraction that simply inspects the angular distance between two adjacent face normals. Although some feature extraction based method as [14] provides robust and convincing results, they still have to compute distance from each vertex of the mesh to the nearest feature point to set global costs. So, result quality of feature extraction based method depends on the quality of extracted feature line. Then, one may spend much time to select an appropriate argument for different input models in different detection scales. The advantage of blow-up weight strategy is that it is quite simple and easy to compute. Instead of tracking an exact feature line, the definition of blow-up weight aims to simulate a uniform point sampling in FS space, and, as we saw in Figs. 2 and 10, this strategy can give a noticeable improvement to a flat FS metric based approach. Those results show that details on the ears of the horse and facial features on the Leopard model may be thrown off instead of remaining during QSlim procedure. It is clearly shown that FS method produces more suitable results for global features especially on head and claws. However, we noticed that some unimportant features like joints and legs have taken excessive patches over
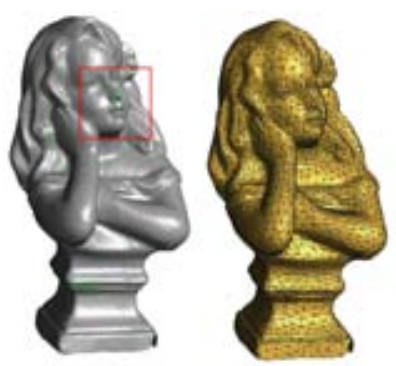

(a)
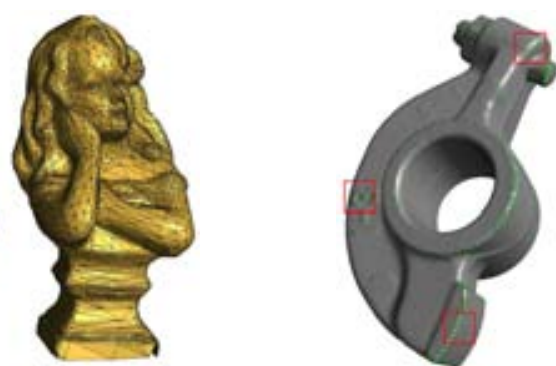
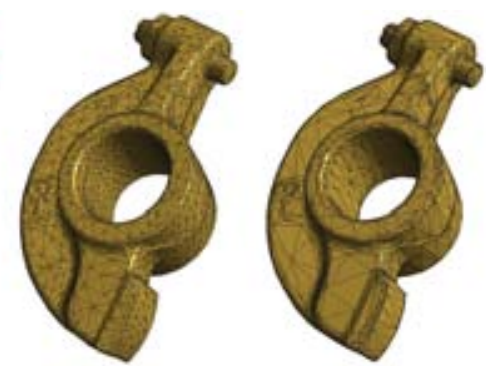

(b)

Fig.9. Contrast of two models between QEM (center) and FS (right). (a) LittleThinking (400K tri. to 7.4K tri.). (b) RockerArm ( $80 \mathrm{~K}$ tri. to $6 \mathrm{~K}$ tri.). Sharp features are tagged in green color. Red cubes are tagged on some feature regions, and the regions are approximated by more triangles than QEM.

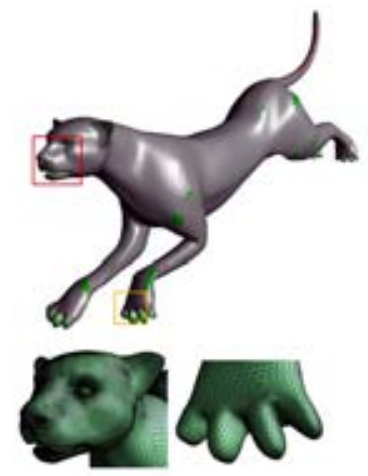

(a)

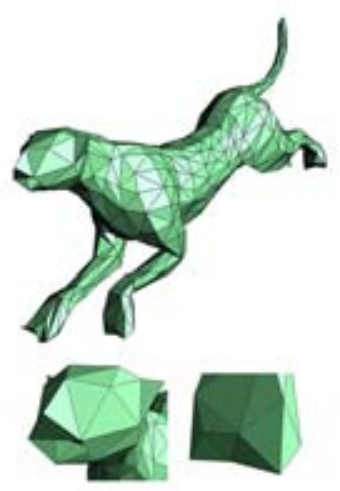

(b)

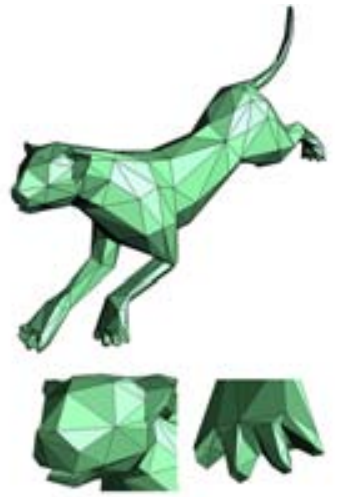

(c)

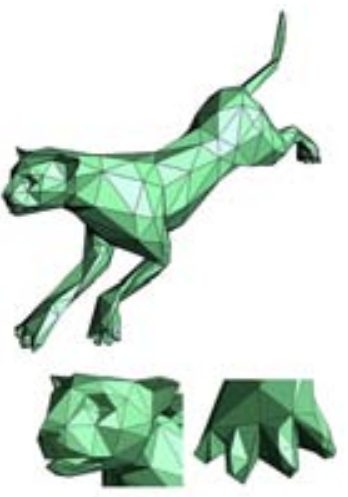

(d)

Fig.10. Leopard model (65024 tri. to 996 tri.) with green (or red) colored feature region. (a) Initial model. (b) Result of QSlim. (c) (d) FS method without and with blow-up, and their zoom in viewer under them, $\omega=0.03$. We see FS method preserves the contour and facial features are completely preserved by FS with blow-up. 
Table 1. Quantization of Max/RMS Errors (The minimum errors are labeled in bold.)

\begin{tabular}{lcccc}
\hline Models (remaining\%) & Types & QSlim & FS & FS with Blow-Up \\
\hline LittleThinking $(1.8 \%)$ & $\mathbb{R}^{3}$ & $0.008774 / \mathbf{0 . 0 0 0 ~ 8 7 4}$ & $\mathbf{0 . 0 0 5 ~ 2 2 6 / 0 . 0 0 0 ~ 9 7 1 ~}$ & - \\
& $\mathbb{R}^{6}$ & $\mathbf{0 . 0 1 2 ~ 6 1 7 / 0 . 0 0 3 ~ 0 9 7}$ & $0.014436 / 0.003109$ & - \\
RockerArm $(7.5 \%)$ & $\mathbb{R}^{3}$ & $0.007141 / 0.000622$ & $\mathbf{0 . 0 0 3 ~ 7 1 7 / 0 . 0 0 0 ~ 5 0 9}$ & - \\
& $\mathbb{R}^{6}$ & $\mathbf{0 . 0 1 6 \mathbf { 1 5 3 } / 0 . 0 0 4 9 7 9}$ & $0.020003 / \mathbf{0 . 0 0 4} \mathbf{4 9 1}$ & $0.026271 / 0.006531$ \\
Horse (6.0\%) & $\mathbb{R}^{3}$ & $0.029360 / 0.005777$ & $\mathbf{0 . 0 1 9 5 7 3 / 0 . 0 0 5 ~ 3 9 2}$ & $0.028869 / \mathbf{0 . 0 1 1 ~ 0 2 4}$ \\
& $\mathbb{R}^{6}$ & $0.026384 / 0.012863$ & $\mathbf{0 . 0 2 2} \mathbf{8 2 7} / 0.011269$ & $0.025460 / 0.004342$ \\
Leopard (1.5\%) & $\mathbb{R}^{3}$ & $0.031569 / 0.007015$ & $\mathbf{0 . 0 1 4 7 9 9 / 0 . 0 0 3 ~ 2 2 3}$ & $\mathbf{0 . 0 2 2} \mathbf{4 3 7} / 0.008740$ \\
HappyBudda(6.5\%) & $\mathbb{R}^{6}$ & $0.033461 / 0.011183$ & $0.022460 / \mathbf{0 . 0 0 8} \mathbf{5 6 9}$ & $0.011453 / 0.001824$ \\
& $\mathbb{R}^{3}$ & $0.012378 / \mathbf{0 . 0 0 1} \mathbf{1 9 5}$ & $\mathbf{0 . 0 1 0 6 2 7} / 0.001891$ & $0.022634 / 0.006240$ \\
\hline
\end{tabular}

simplified model rather than assigned on the head (Fig.2 bottom), because joints may consist of many small triangles in original model so that the geometric variance near the joints may be larger than which is on the head. Similarly, eyes were also eliminated on the simplified Leopard model. Although for textured models, these results derived by standard FS method are sufficient for most applications. One may still prefer to get a more compact abstraction that preserve most of main facial features. To achieve this goal, we may introduce the blow-up weight strategy. Fig.2(d) and Fig.10(d) show the results produced by weighted FS method. For the same number of remaining triangles and blow-up arguments, facial features are approximated by more patches but little on legs and tails. Visual effects for both models got good enhancements. These results have demonstrated our weight strategy is effective and easy to use. It is useful when users have to face to a large database.

It is noticed that having more triangles on features means remaining less on the planar regions. The more features are remained, the larger the error will be introduced in planar regions. On the other hand, QEM method uses a geometric error-driven greedy optimiza-

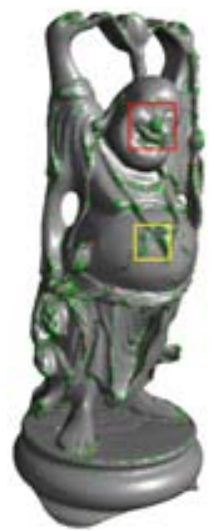

(a)

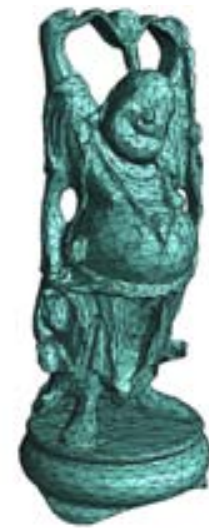

(b)

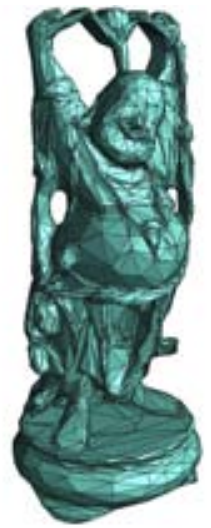

(c)

tion, all the target vertex is derived under least-squared sense, so that to deal with RMS (Root Mean Squared) errors, FS method does not provide remarkable improvement. Table 1 lists error comparisons for all example models used in this paper, with its remaining rate correspondingly. We see that for FS methods, most RMS errors are smaller, but QSlim outperforms occasionally. On the other hand, for weighted FS method, though most of remaining feature points are preserved, RMS error may still exceed QSlim because the planar regions are approximated by the triangles that are even larger than a flat FS method. But we claim that as FS methods get much lower maximum errors, FS methods make much better outputs for visual effect. So these results are much more useful than those derived by QSlim. Another example of HappyBudda model is shown in Fig.11. The corresponding errors are also listed in Table 1. We see that although QSlim outperforms our method on RMS error, features (marked as green color in Fig.11(a)) are preserved much better by FS. When the blow-up weight is applied, details of remaining features are superior to flat FS method.

It is notable that reasonable arguments for feature sensitivity will give different distributions of triangles

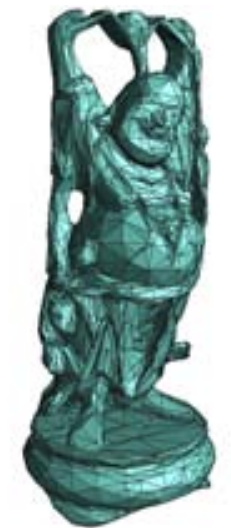

(d)

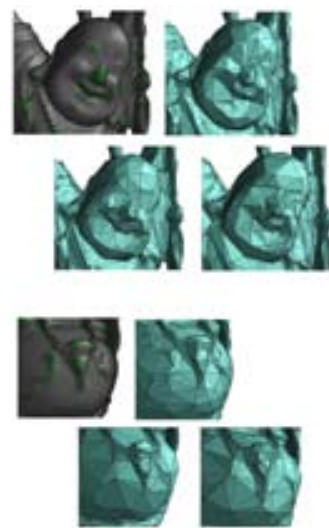

(e)

Fig.11. HappyBudda model (123056 tri. to 8032 tri.) with feature region colored in green or red. (a) Initial mesh. (b) QSlim result. (c) (d) FS method without and with blow-up. Their zoom in images are shown in (e) in the order. 

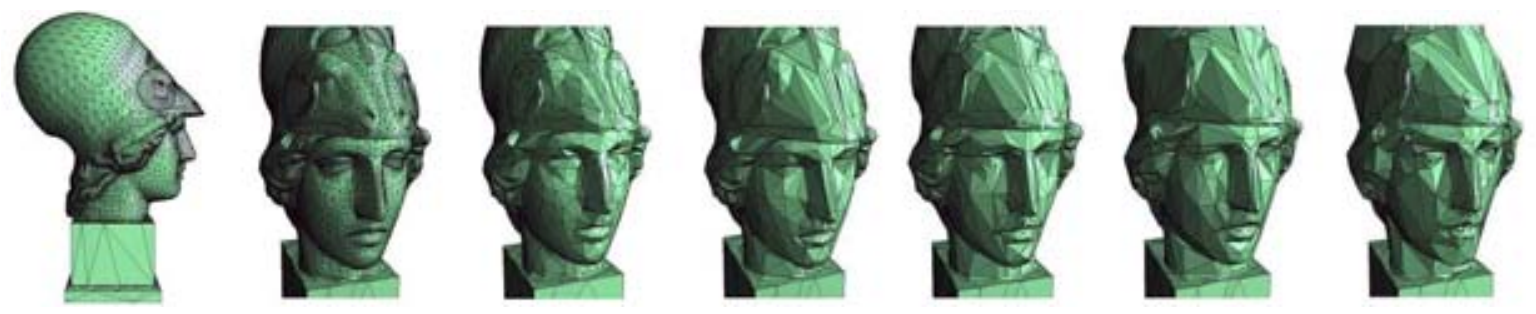

Fig.12. Simplifying Ateneam model with various feature sensitivity arguments, 15104 to 1992 tri.: All simplified models is derived by FS method without blow-up, $\omega$ is set as $0,0.01,0.03,0.1,0.3$. If $\omega$ is set to 0 , the result is similar to the previous QEM method. When $\omega$ is upgraded, more triangles are retained on the sharp edges but distortion is also occurred at the planar regions.

for simplified mesh. Fig.12 shows a series of simplified Ateneam models with different $w$ in ascending order. We see the facial features are approximated by further patches under larger weights. However, large weight may cause too large distortions on planar regions. When $w$ is upgraded to 0.3 , though the details of eyes and mouth are preserved well, the overall appearance of facial form becomes unacceptable.

Although our inception is focused on the accuracy but is not efficiency during simplification, we are still concerned about performance. A direct speculation is that, as FS methods take more dimensions into account, and iterate at each step, we have to cost more time than QEM method. We implemented our method and QSlim is also integrated into the same testing framework, comparing the performance for both methods on a laptop with Intel Duo $2.5 \mathrm{GHz}$ processor and $2 \mathrm{~GB}$ main memory. Table 2 shows the comparison results for each model used in this paper. Our approach costs around double times than QSlim in most cases. This is an limitation of FS method that there exists a tradeoff between appearance, speed and interactivity, but it is still faster than some other appearance preserving based method like [15], that is mentioned as three to twenty times slower than QEM. For fair comparison, we do not introduce any special scheme for acceleration, so there are quite a lot strategies which can be employed for further improvement, e.g., using vertex heap rather than edge heap ${ }^{[18]}$. The performance of FS method is also related to the input quality, for the reason that a complex model may bring more iteration steps.

Table 2. Time Comparison (in ms)

\begin{tabular}{lrrr}
\hline Models & No. Faces & QSlim & \multicolumn{1}{c}{ FS } \\
\hline Horse & 10024 & 180 & 312 \\
Leopard & 65024 & 1224 & 2612 \\
RockerArm & 80354 & 1650 & 3129 \\
HappyBudda & 123056 & 2663 & 4922 \\
Lucy & 237278 & 5399 & 9871 \\
LittleThinking & 401985 & 8249 & 17463 \\
\hline
\end{tabular}

\section{Conclusions and Future Work}

We have presented a new simplification algorithm based on FS metric. According to the constraint conditions, we construct error metric by using the sum of the squared distance from a $6 \mathrm{D}$ point to its corresponding two-dimensional hyperplane rather than in $\mathbb{R}^{3}$. Moreover, we employed a constrained optimization to acquire contraction target point which satisfies the geometric constraint on normal directions, this strategy may also be combined with other normal-geometry related cases. Experimental results demonstrated that this algorithm can preserve feature more precisely under the global geometry, and then naturally retain more patches on the feature regions without special feature recognitions during the simplification process.

Taking the advantage of blow-up phenomenon in FS space, our method can produce more suitable result and high visual quality on feature regions.

However, as we have known that the tradeoff between efficiency and accuracy has become the bottleneck to simplification, feature sensitive simplification with the high-performance computing technology may become a significative research topic.

\section{References}

[1] Heckbert P, Garland M. Survey of polygonal surface simplification algorithms. In SIGGRAPH 1997 Course Notes: Multiresolution Surface Modeling, 1997.

[2] Garland M, Heckbert P. Surface simplification using quadric error metrics. In Proc. SIGGRAPH, Los Angeles, USA, Aug. 3-8, 1997, pp.209-216.

[3] Hoppe H. New quadric metric for simplifying meshes with appearance attributes. In Proc. the 10th IEEE Visualization Conference, San Francisco, USA, Oct. 24-29, 1999, pp.59-66.

[4] Yan J, Shi P, Zhang D. Mesh simplification with hierarchical shape analysis and iterative edge contraction. IEEE Transactions on Visualization and Computer Graphics, 2004, 10(2): 142-151.

[5] Jong B S, Teng J L, Yang W H. An efficient and low-error mesh simplification method based on torsion detection. The Visual Computer, 2005, 22(1): 56-67.

[6] Kimmel R, Malladi R, Sochen N. Image as embedded maps and minimal surfaces: Movies, color, texture and volumetric medical images. International Journal of Computer Vision, 2000, 39(2): 111-129. 
[7] Lai Y K, Zhou Q Y, Hu S M, Wallner J, Pottmann H. Robust feature classification and editing. IEEE Transactions on Visualization and Computer Graphics, 2007, 13(1): 34-45.

[8] Lai Y K, Hu S M, Pottmann H. Surface fitting based on a feature sensitive parameterization. Computer-Aided Design, 2006, 38(7): 800-807.

[9] Lai Y K, Zhou Q Y, Hu S M, Martin R R. Feature sensitive mesh segmentation. In Proc. ACM Symposium on Solid and Physical Modeling, Cardiff, UK, June 6-8, 2006, pp.17-25.

[10] Cohen J, Olano M, Manocha D. Appearance-preserving simplification. In Proc. the 25th Annual Conference on Computer Graphics and Interactive Techniques, Orlando, USA, July 19-24, 1998, pp.115-122.

[11] Kho Y, Garland M. User-guided simplification. In Proc. ACM Symposium on Interactive $3 D$ Graphics, Monterey, USA, April 27-30, 2003, pp.123-126.

[12] Garland M, Zhou Y. Quadric-based simplification in any dimension. ACM Transactions on Graphics, 2005, 24(2): 209239.

[13] Lindstrom P, Turk G. Image-driven simplification. ACM Transactions on Graphics, 2000, 19(3): 204-241.

[14] Yoshizawa S, Belyaev A G, Seidel H P. Fast and robust detection of crest lines on meshes. In Proc. ACM Symposium on Solid and Physical Modeling, Cambridge, USA, June 13-15, 2005, pp.13-15.

[15] Cohen-Steiner D, Alliez P, Desbrun M. Variational shape approximation. In Proc. ACM SIGGRAPH, Los Angeles, USA, Aug. 8-12,, 2004 pp.905-914.

[16] Bian Z, Hu S M, Martin R R. Evaluation for small visual difference between conforming meshes on strain field. Journal of Computer Science and Technology, 2009, 24(1): 65-75.

[17] Cignoni P, Rocchini C, Scopigno R. Metro: Measuring error on simplified surfaces. Computer Graphics Forum, 1998, 17(2): 167-174

[18] Hussain M. Efficient simplification methods for generating high quality LODs of 3D meshes. Journal of Computer Science and Technology, 2009, 24(3): 604-inside back cover.

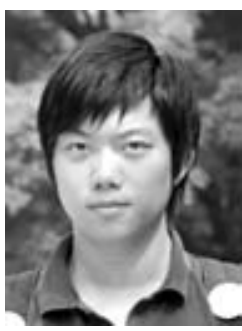

Jin Wei is a Master candidate at the Department of Computer Science and Technology, Tsinghua University. His research interests include digital geometry processing, video processing, and computational camera. He is a student member of China Computer Federation and ACM.

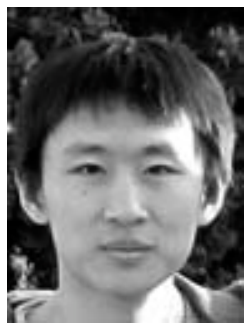

Yu Lou is currently a Master candidate in the Department of Computer Science, Stanford University. His research interests include computer graphics, mesh processing, and image processing. He is a student member of ACM. 